\title{
Pediatric Radiopharmaceutical Administration: Harmonization of the 2007 EANM Paediatric Dosage Card (Version 1.5.2008) and the 2010 North American Consensus guideline
}

\author{
Michael Lassmann • S. Ted Treves
}

Published online: 4 June 2014

(C) Springer-Verlag Berlin Heidelberg 2014

Dear Sir,

We write in reply to Dr. Metrard's letter to the editor [1]. We appreciate his interest in the common EANM and SNNMI harmonization document which has been approved recently by both societies [2]. In his letter Dr. Metrard raises the question why, in the harmonized dosage card, the recommended activities for FDG-PET Torso are higher for the EANM dosage card as compared to the activities range between 3.7 MBq/kg and 5.2 MBq/kg provided by the 2010 North American Consensus guidelines [3] or the even lower activity of $3.2 \mathrm{MBq} / \mathrm{kg}$ proposed by Alessio et al. [4].

As there still is a considerable variety of PET/CT scanners in operation, and the protocols for FDG-PET scanning are still variable between different institutions, the values given in the harmonization document represent, for Europe, upper limits of the activities to be administered. Of course, as has been stated in the addendum to the previous version of the EANM dosage card [5], the state of the art acquisition techniques and the sensitivity of the PET/CT scanner in the different institutions should be taken into account when lowering the activity to be administered. A diagnostic procedure that needs to be repeated because of insufficient image quality causes unnecessary radiation exposure; it also might prevent finding a correct diagnosis within a short timeframe.

\section{Lassmann $(\bowtie)$}

Department of Nuclear Medicine, University Würzburg,

97080 Würzburg, Germany

e-mail: lassmann_m@ukw.de

\section{S. T. Treves}

Harvard Medical School, Nuclear Medicine and Molecular Imaging,

Brigham and Women's Hospital, Boston, MA 02115, USA

e-mail: streves@partners.org

S. T. Treves

e-mail: ted_treves@HMS.harvard.edu
Should more evidence for further reducing the activities without impeding image quality for more scanners become available, and more uniform protocols for FDG-PET scans in children are developed, a further reduction of the administered activities might be recommended in future versions of the dosage card.

\section{References}

1. Metrard G. New EANM paediatric dosage card: optimization of F-18 FDG administered activities. Eur J Nucl Med Mol Imaging. 2014. doi: 10.1007/s00259-014-2816-5.

2. Lassmann M, Treves ST, EANM. SNMMI Paediatric Dosage Harmonization Working Group. Paediatric radiopharmaceutical administration: harmonization of the 2007 EANM paediatric dosage card (version 1.5.2008) and the 2010 North American consensus guidelines. Eur J Nucl Med Mol Imaging. 2014;41:1036-41.

3. Gelfand MJ, Parisi MT, Treves ST. Pediatric Nuclear Medicine Dose Reduction Workgroup. Pediatric radiopharmaceutical administered doses: 2010 North American consensus guidelines. J Nucl Med. 2011;52:318-22.

4. Alessio AM, Sammer M, Phillips GS, Manchanda V, Mohr BC, Parisi MT. Evaluation of optimal acquisition duration or injected activity for pediatric 18F-FDG PET/CT. J Nucl Med. 2011;52:1028-34.

5. Lassmann M, Biassoni L, Monsieurs M, Franzius C. The new EANM paediatric dosage card: additional notes with respect to F-18. Eur J Nucl Med Mol Imaging. 2008;35:1666-8.cpf 